\title{
Semiperiodo de la literatura científica en Biblioteconomía y Documentación y la influencia de los aspectos temáticos
}

\author{
J. A. Martínez-Comeche \\ M.J García Martín \\ J. Horcas Pulido \\ M. de la Peña Martín \\ R.I. Pérez Donoso *
}

Artículo recibido:

9 de noviembre de 2009.

Artículo aceptado:

6 de septiembre de 2010.

\section{RESUMEN}

Este trabajo presenta el cálculo del semiperiodo o vida media de las referencias en 28 revistas españolas y anglosajonas de Biblioteconomía y Documentación siguiendo la fórmula de Burton y Kebler, durante el año 2006. Se analiza posteriormente la posible relación con los contenidos presentes en las revistas, y se extraen conclusiones sobre qué temas nucleares del área imponen básicamente dicho semiperiodo, dando lugar a lo que puede considerarse como el semiperiodo de la literatura científica en Biblioteconomía y Documentación, y señalando qué temas tienden a aumentar o disminuir dicho semiperiodo cuando están presentes en

* Los autores pertenecen a la Universidad Complutense de Madrid, España. (contacto: juaamart@pdi.ucm.es)

INVESTIGACIÓN BiBLIOTECOLÓGICA, Vol. 24, Núm. 51, mayo/agosto, 2010, México, ISSN: 0187-358X. pp. 195-207 
una determinada revista. De esta forma el semiperiodo podría considerarse un indicio del contenido temático de la revista.

Palabras clave: Semiperiodo, Biblioteconomía y Documentación, Contenidos temáticos.

\begin{abstract}
The half-life of scientific literature in library and information science and the influence of thematic issues

J.-A. Martínez-Comeche; M. J. García Martín; J. Horcas

Pulido; M. de la Peña Martín and R. I. Pérez Donoso

Following the formula developed by Burton and Kebler, this paper presents a calculation of the half-life of bibliographical references appearing in twenty-eight English- and Spanish-language Library and Information Science journals in the year 2006. An analysis of the relationship of this phenomenon to content is carried out in order to identify the nuclear topic areas that in turn determine this half-life. Moreover, the paper provides an assessment of those subject areas for each of the journals studied that tend to lengthen or shorten the half-life of a given bibliographic reference. As such, the bibliographic half-life may be considered an indicator of the thematic inclination of a given journal.
\end{abstract}

Keywords: half-life, Library and Information Science, thematic content

\title{
I. INTRODUCCIÓN
}

E1 1 l objetivo del presente artículo es calcular el semiperiodo o vida media de la literatura científica en Biblioteconomía y Documentación en 2006 a través de las revistas del área, esto es, la antigüedad media de los artículos citados en dichas revistas durante el año 2006 [Journal Citation Reports, Cited Half-Life]. Siguiendo con el ejemplo de Journal Citation Reports, en 2001 la revista Crystal Research and Technology presenta un semiperiodo de las referencias de 7. Ello significa que los artículos publicados en Crystal Research and Technology incluyeron referencias a otros artículos, la mitad de las cuales 
se situaban cronológicamente en los 7 años anteriores; esto es, entre 1995 y 2001 (ambos inclusive).

En este trabajo calculamos el semiperiodo o vida media de un total de 28 revistas del área, la mitad redactadas en inglés y la mitad españolas, que presentaban un mejor factor de impacto en 2005. Los distintos resultados obtenidos por las revistas no implican resultados mejores o peores para ellas. En primera instancia, el cálculo del semiperiodo o vida media puede ser de utilidad para los bibliotecarios de cara a la toma de decisiones en relación a la gestión de colecciones de revistas y en concreto en cuanto a su archivado, pero nosotros también hemos analizado la posible relación que presenta el semiperiodo con los contenidos específicos de las revistas, y hemos extraído conclusiones sobre qué temas nucleares modulan básicamente el semiperiodo hacia el valor mayoritario en las revistas analizadas, dando lugar a lo que puede considerarse como el semiperiodo de la literatura científica de la Biblioteconomía y Documentación, y a aquellos temas que tienden a aumentar o disminuir esta vida media cuando están presentes en una revista.

\section{Metodología}

Para analizar el semiperiodo del área a través del análisis de las citas producidas en las revistas de Biblioteconomía y Documentación y su relación con los contenidos temáticos de ellas, tanto en inglés como en español, partimos de una amplia muestra compuesta por 28 revistas, la mitad de ellas españolas y la otra mitad redactadas en inglés. La elección se efectuó en función de su mayor factor de impacto [Thomson Reuters. Introducing the impact factor], con el ánimo de contar con las revistas de mayor importancia relativa en nuestro campo de conocimiento.

Para las revistas españolas se utilizó la lista proporcionada por IN-RECS (índice de impacto de las revistas españolas de Ciencias Sociales) para el área de Biblioteconomía y en relación al año 2005 [IN-RECS, Biblioteconomía. Revistas ordenadas por Índice de impacto]. Si se consulta actualmente dicha fuente se observará que la población de revistas ha aumentado de 22 en 2006 hasta las 26 actuales, por lo que existen diferencias entre las revistas situadas en las primeras posiciones entonces y en la actualidad. Lógicamente reproducimos aquí la lista tal como se obtuvo en 2006, fecha del inicio del trabajo: 
Tabla1. Revistas españolas de Biblioteconomía en 2005 ordenadas por Índice de impacto

\begin{tabular}{|l|c|}
\hline \multicolumn{1}{|c|}{ Título de revistas españolas } & Abreviatura empleada \\
\hline $\begin{array}{l}\text { Cybermetrics: International Journal of Scientometrics, Informetrics and } \\
\text { Bibliometrics }\end{array}$ & Cybermetrics \\
\hline El Profesional de la Información & EPI \\
\hline BID: Textos Universitaris de Biblioteconomía i Documentació & BID \\
\hline Revista Española de Documentación Científica & RCDC \\
\hline Documentación de las Ciencias de la Información & AD \\
\hline Anales de Documentación & BAAB \\
\hline Boletín de la Asociación Andaluza de Bibliotecarios & BANABAD \\
\hline Boletín de la ANABAD & Item \\
\hline Item. Revista de Biblioteconomía i Documentació & RGID \\
\hline Revista General de Información y Documentación & EyB \\
\hline $\begin{array}{l}\text { Educación y Biblioteca. Revista Mensual de Documentación y Recursos } \\
\text { Didácticos }\end{array}$ & AABADOM \\
\hline $\begin{array}{l}\text { Aabadom: Boletín de la Asociación Asturiana de Bibliotecarios, Archiveros, } \\
\text { Documentalistas y Museólogos }\end{array}$ & BIBLIODOC \\
\hline Bibliodoc: Anuari de Biblioteconomia, Documentació i Informació & BILDUMA \\
\hline Bilduma: Revista del Servicio de Archivo del Ayuntamiento de Errenteria & CDM \\
\hline Cuadernos de Documentación Multimedia & \\
\hline
\end{tabular}

En esta lista figuran 15 revistas en lugar de las 14 inicialmente previstas. Ello se debe a que Aabadom, el Boletín de la Asociación Asturiana de Bibliotecarios, Archiveros, Documentalistas y Museólogos, no publicó finalmente un volumen correspondiente al año 2006, por lo que decidimos sustituir dicha revista por la siguiente en la lista, los Cuadernos de Documentación Multimedia. De igual forma, Bilduma no publicó el volumen correspondiente al año 2006. Puestos al habla con los responsables de la misma, se nos señaló la posibilidad de que no volviese a publicarse un número posterior, por lo que en este caso se optó por emplear el volumen correspondiente al año 2005.

Para las revistas en inglés se consultó la lista proporcionada por JCR (Journal Citation Reports) correspondiente al área Information Science \& Library Science y al año 2005 [Journal Citation Reports, Social Science Edition. 2005. Journal Summary List sorted by impact factor]:

Tabla 2. Revistas en inglés sobre Information \& Library Science en 2005 (por factor de impacto).

\begin{tabular}{|l|c|}
\hline \multicolumn{1}{|c|}{ Título de revistas redactadas en inglés } & Abreviatura empleada \\
\hline MIS Quarterly & MISQ \\
\hline Journal of the American Medical Informatics Association & JAMIA \\
\hline Annual Review of Information Science and Technology & ARIST \\
\hline Information Systems Research & ISR \\
\hline Scientometrics & Scientometrics \\
\hline Journal of the American Society for Information Science and Technology & JASIST \\
\hline International Journal of Geographical Information Science & IJGEOIS \\
\hline
\end{tabular}




\begin{tabular}{|l|c|}
\hline Journal of Information Technology & JIT \\
\hline Information \& Management & IM \\
\hline Journal of Management Information Systems & JMIS \\
\hline College \& Research Libraries & CRL \\
\hline Journal of the Medical Library Association & JMLA \\
\hline Information Processing \& Management & IPM \\
\hline Information Society & IS \\
\hline
\end{tabular}

Elegidas las revistas se procedió a recopilar los artículos publicados durante 2006 en ellas, y posteriormente a anotar la fecha de publicación de las referencias citadas en todos los artículos, desde 2006 hacia atrás. Han sido escasas las referencias que no incluían una fecha, por lo que su exclusión de la muestra no afecta los resultados obtenidos. De igual forma, si una referencia abarcaba varias fechas, se consideró la fecha más reciente como la de publicación del documento citado. Siguiendo este mismo criterio, cuando la cita señalaba "in press" o "en prensa", se consideró la fecha de publicación más reciente posible, esto es, 2006. Con tales datos se pueden calcular diversos semiperiodos, desde el correspondiente a cada revista hasta el global correspondiente al conjunto de todas ellas, pasando por el semiperiodo del conjunto de las revistas españolas o de las revistas redactadas en inglés.

Es posible emplear diversos métodos estadísticos para calcular el envejecimiento de la literatura científica [Ruiz Baños, Bailón-Moreno, 1997], de entre los cuales hemos elegido el semiperiodo, vida media o semi-vida conforme a la fórmula inicialmente propuesta por Burton y Kebler [Burton, Kebler, 1960]:

$$
y=1-a / e^{x}+b / e^{2 x}-
$$

donde:

$\mathrm{a}+\mathrm{b}=1$

$\mathrm{y}=$ porcentaje acumulado de citas expresado como una cifra decimal entre 0 y 1

$\mathrm{x}=$ tiempo expresado en décadas

el semiperiodo es el valor de $\mathrm{x}$ correspondiente a $\mathrm{y}=0.5$

Posteriormente se ha procedido a efectuar un análisis temático de los artículos que componen la muestra utilizada. Para ello se ha partido del Tesauro de Biblioteconomía y Documentación del Centro de Información y Documentación Científica (CINDOC) de España [CINDOC. Tesauro de Biblioteconomía y Documentación]. Inicialmente se procedió a imponerle a cada artículo hasta tres descriptores de cualquier nivel de profundidad procedentes 
del tesauro, para posteriormente, reunidos todos los autores, decidir conjuntamente un único descriptor temático por artículo de entre los 12 descriptores del primer nivel jerárquico del tesauro:

- Archivística (abreviatura: A)

- Biblioteconomía (abreviatura: B)

- Ciencias y técnicas auxiliares (abreviatura: C)

- Estudios métricos de la información (abreviatura: E)

- Fuentes de información (abreviatura: F)

- Lenguajes y lingüística (abreviatura: L)

- Museología (abreviatura: M)

- Proceso documental (abreviatura: PD)

- Profesionales de la información y usuarios (abreviatura: PU)

- Sociedad de la información (abreviatura: S)

- Tecnologías de la información y las comunicaciones (abreviatura: T)

- Unidades de información (abreviatura: U)

\section{Análisis de resultados}

Calculados los semiperiodos correspondientes a cada revista en el año 2006, se han obtenido los siguientes resultados:

Tabla 3. Semiperiodo de las revistas de la muestra en 2006

\begin{tabular}{|l|c|c|c|}
\hline \multicolumn{1}{|c|}{ Revista } & Semiperiodo & Revista & Semiperiodo \\
\hline Arist & 7.75447 & Ad & 8.94333 \\
\hline Crl & 5.26502 & Baab & 3.00521 \\
\hline Ijgeois & 6.83198 & Banabad & 4.34411 \\
\hline Im & 7.13851 & Bibliodoc & 3.36867 \\
\hline Ipm & 5.80891 & Bid & 4.08955 \\
\hline Is & 6.13933 & Bilduma & 15.48013 \\
\hline Isr & 7.69595 & Cdm & 12.23441 \\
\hline Jamia & 4.52388 & Cybermetrics & 3.67902 \\
\hline Jasist & 7.16914 & Dci & 9.00419 \\
\hline Jit & 8.42547 & Epi & 3.97586 \\
\hline Jmis & 7.96880 & Eyb & 10.40399 \\
\hline Jmla & 4.48027 & Item & 7.88279 \\
\hline Misq & 9.03740 & Redc & 5.39729 \\
\hline Scientometrics & 8.38166 & Rgid & 12.35928 \\
\hline
\end{tabular}

Considerando globalmente todos los artículos de la muestra, ya sean en español o en inglés, se obtiene un semiperiodo global de 6.91 años. Dado el 
volumen de la muestra (un total de 41143 referencias válidas), puede considerarse ésta una buena aproximación sobre el semiperiodo de la literatura científica en Biblioteconomía y Documentación.

Considerando aisladamente las referencias presentes en las revistas del área anglosajona (37525) se obtiene un semiperiodo de 6.97 años. Análogamente, observando exclusivamente las referencias incluidas en las revistas españolas (3618), se obtiene un valor para el semiperiodo de 6.25 años. Aunque existe una gran diferencia entre el número de referencias recopiladas en inglés (37525) y el número de referencias recopiladas en español (3618), los datos muestran que el envejecimiento de las referencias en las revistas españolas tiende a ser ligeramente más rápido (esto es, las citas tienden a ser más actuales) que el envejecimiento observado en las referencias incluidas en las revistas del área anglosajona (esto es, entre las citas se hallan referencias un poco más antiguas). Estos datos confirman la tendencia puesta de manifiesto en estudios anteriores, aunque de manera más moderada [Ruiz Baños, Jiménez-Contreras, 1996].

Otro aspecto destacado que resalta de la comparación entre las revistas españolas y anglosajonas es la mayor variabilidad del semiperiodo en el caso de las revistas españolas sobre Biblioteconomía y Documentación. Si el semiperiodo de las revistas en inglés oscila entre 9.04 y 4.48 años (lo que implica un rango de 4.56 años), el rango en el que se mueven los semiperiodos de las revistas españolas del área se eleva a 12.47 años (entre un máximo de 15.48 y un mínimo de 3.01 años). A nuestro juicio, este hecho se puede explicar acudiendo a la influencia de los aspectos temáticos en el valor del semiperiodo. Dando por cierta dicha relación, que trataremos de mostrar a continuación, el mayor rango en el valor del semiperiodo en el caso de las revistas españolas se debería a una mayor concentración de artículos sobre una temática concreta en dichas revistas. Por ejemplo, si una revista específica acumula un porcentaje mayoritario de artículos sobre una temática con un envejecimiento menor, obtendremos una revista que, perteneciendo al mismo área de la Biblioteconomía y Documentación, presenta un semiperiodo muy elevado y alejado de la media del área. De igual forma, el rango menor en el caso de las revistas del área anglosajona se explicaría aduciendo que cada una de dichas revistas presenta, en general, una diversidad temática mayor, lo que se refleja en semiperiodos menos alejados de la media.

Para comprobar esta influencia de los aspectos temáticos en el valor final del semiperiodo, resumimos en la siguiente tabla los porcentajes (tanto por uno) hallados de cada uno de los 12 descriptores temáticos en cada una de las revistas de la muestra empleada: 
Tabla 4. Porcentaje de temas presentes en las revistas en inglés en 2006

\begin{tabular}{|l|c|c|c|c|c|c|c|c|c|c|c|c|}
\hline & A & B & C & E & F & L & M & PD & PU & S & T & U \\
\hline Arist & 0 & 0 & 0.23 & 0 & 0 & 0.08 & 0 & 0.31 & 0 & 0 & 0.38 & 0 \\
\hline Crl & 0 & 0.19 & 0 & 0.16 & 0 & 0 & 0 & 0 & 0.44 & 0 & 0.12 & 0.09 \\
\hline Ijgeoi & 0 & 0 & 0 & 0.03 & 0.03 & 0.03 & 0 & 0.12 & 0 & 0.04 & 0.75 & 0 \\
\hline Im & 0 & 0 & 0.08 & 0.04 & 0 & 0.01 & 0 & 0.04 & 0.26 & 0.06 & 0.51 & 0 \\
\hline Ipm & 0 & 0 & 0 & 0.18 & 0.01 & 0.04 & 0 & 0.38 & 0.04 & 0.01 & 0.34 & 0 \\
\hline Is & 0 & 0 & 0 & 0 & 0 & 0 & 0 & 0.05 & 0.22 & 0.43 & 0.30 & 0 \\
\hline Isr & 0 & 0 & 0.13 & 0.04 & 0 & 0 & 0 & 0.08 & 0 & 0.08 & 0.67 & 0 \\
\hline Jamia & 0 & 0.04 & 0.07 & 0.04 & 0 & 0.05 & 0 & 0.06 & 0.13 & 0.01 & 0.60 & 0 \\
\hline Jasist & 0 & 0.01 & 0.05 & 0.20 & 0.01 & 0.03 & 0 & 0.19 & 0.08 & 0.03 & 0.38 & 0.02 \\
\hline Jit & 0 & 0 & 0.04 & 0.04 & 0 & 0 & 0 & 0 & 0 & 0.12 & 0.80 & 0 \\
\hline Jmis & 0 & 0 & 0.09 & 0.04 & 0.02 & 0.02 & 0 & 0.08 & 0 & 0.02 & 0.73 & 0 \\
\hline Jmla & 0 & 0.06 & 0.09 & 0.15 & 0.06 & 0.15 & 0 & 0.06 & 0.27 & 0 & 0.13 & 0.03 \\
\hline Misq & 0 & 0 & 0.11 & 0 & 0.07 & 0 & 0 & 0.02 & 0.02 & 0 & 0.78 & 0 \\
\hline Scient & 0 & 0 & 0.03 & 0.80 & 0.03 & 0.01 & 0 & 0.03 & 0.03 & 0.02 & 0.05 & 0 \\
\hline
\end{tabular}

Tabla 5. Porcentaje de temas presentes en las revistas españolas en 2006

\begin{tabular}{|l|c|c|c|c|c|c|c|c|c|c|c|c|}
\hline & A & B & C & E & F & L & M & PD & PU & S & T & U \\
\hline Ad & 0 & 0.15 & 0.15 & 0.08 & 0.08 & 0.08 & 0 & 0 & 0.31 & 0 & 0.15 & 0 \\
\hline Baab & 0 & 0.67 & 0 & 0 & 0 & 0 & 0 & 0 & 0.33 & 0 & 0 & 0 \\
\hline Bana & 0.20 & 0.20 & 0.22 & 0 & 0.03 & 0 & 0.03 & 0 & 0.03 & 0.09 & 0.14 & 0.06 \\
\hline Bibli & 0 & 0.29 & 0.43 & 0 & 0 & 0.14 & 0 & 0 & 0 & 0 & 0 & 0.14 \\
\hline Bid & 0 & 0.06 & 0.13 & 0.06 & 0 & 0 & 0 & 0.06 & 0.19 & 0 & 0.31 & 0.19 \\
\hline Bildu & 0 & 0 & 1 & 0 & 0 & 0 & 0 & 0 & 0 & 0 & 0 & 0 \\
\hline Cdm & 0 & 0 & 0 & 0 & 0.75 & 0 & 0 & 0 & 0 & 0.25 & 0 & 0 \\
\hline Ciber & 0 & 0 & 0 & 0.85 & 0 & 0 & 0 & 0 & 0 & 0 & 0.15 & 0 \\
\hline Dci & 0.13 & 0.06 & 0.25 & 0.06 & 0.19 & 0.06 & 0 & 0 & 0.06 & 0.06 & 0.13 & 0 \\
\hline Epi & 0.04 & 0.05 & 0.02 & 0.19 & 0.05 & 0 & 0 & 0.04 & 0.04 & 0.09 & 0.43 & 0.05 \\
\hline Eyb & 0 & 0.02 & 0.04 & 0 & 0.07 & 0 & 0 & 0.02 & 0.54 & 0 & 0.09 & 0.22 \\
\hline Item & 0 & 0.13 & 0.13 & 0.13 & 0 & 0 & 0 & 0.13 & 0.13 & 0 & 0.35 & 0 \\
\hline Redc & 0.06 & 0 & 0.06 & 0.49 & 0.06 & 0 & 0 & 0.06 & 0 & 0 & 0.21 & 0.06 \\
\hline Rgid & 0.09 & 0.05 & 0.35 & 0 & 0.05 & 0.09 & 0 & 0.05 & 0.05 & 0.09 & 0.05 & 0.13 \\
\hline
\end{tabular}

De las tablas precedentes, y en un análisis inicial, podemos inferir que existen ciertos temas cuya presencia mayoritaria en ciertas revistas provoca que el semiperiodo de tales revistas se mantenga en un margen cercano al promedio del área (entre 7 y 8). Es, por ejemplo, el caso de Scientometrics, con un 80\% de artículos sobre Estudios métricos y un semiperiodo de 8.38. Jasist, por su parte, incluye en su volumen un $40 \%$ de artículos sobre Tecnologías de la información, un 20\% sobre Estudios métricos y otro 20\% sobre Proceso documental, con lo que obtiene un semiperiodo de 7.17. Arist, por su parte, también presenta un 40\% de artículos sobre Tecnologías de la información y un 30\% sobre Proceso documental, con lo que concuerda con el semiperiodo de 7.75 que posee. 
En cambio la presencia porcentualmente importante de temas como Profesionales de la información y usuarios provoca que el semiperiodo de la revista tienda a disminuir. Es el caso de $\mathrm{Crl}$, con un $44 \%$ de artículos sobre dicha temática y un semiperiodo de 5.27. Jmla, por su parte, contiene un primer bloque importante por el número de artículos $(27 \%)$, relativo a Profesionales y usuarios, poseyendo un semiperiodo de 4.48. Jamia puede también presentarse como ejemplo, pues después de obtener un $60 \%$ de artículos sobre Tecnologías, e incluir un 13\% de artículos sobre Profesionales y usuarios, el valor de su semiperiodo fue de 4.52 .

En un primer análisis otro tema que parece facilitar la disminución del semiperiodo es Bibliotecomía. Aunque el tema es poco frecuente en la muestra utilizada, es el segundo bloque temático en importancia (29\%) en la revista Bibliodoc, al presentar dicha revista un semiperiodo de 3.37. De igual forma, la presencia de este aspecto temático en un $19 \%$ en la revista $C r l$ parece favorecer su semiperiodo de 5.27.

De los 12 descriptores temáticos empleados, el correspondiente a Ciencias auxiliares es el menos específico (abarca desde Derecho hasta Historia, pasando por las técnicas auxiliares), lo que provoca un comportamiento variable que depende de qué aspecto temático concreto se trate en cada caso. Así, puede contribuir a disminuir el semiperiodo de la revista cuando alude a la Ciencia de la Documentación. Es el caso de Banabad, donde el primer bloque temático en cantidad de artículos corresponde a Ciencias auxiliares, y en concreto a la Ciencia de la Documentación, con un 22\% de artículos, y el segundo bloque temático en importancia corresponde a la Biblioteconomía, que resultó con un semiperiodo de 4.34.

En cambio cuando Ciencias auxiliares alude a Historia, Epigrafía o Numismática, por ejemplo, y su presencia en la revista es relevante, claramente contribuye a aumentar el semiperiodo resultante. El caso más claro lo tenemos en Bilduma, pues la totalidad del volumen empleado es de carácter histórico y presenta el semiperiodo más alto (15.48) de toda la muestra. Otro ejemplo destacado es Rgid, donde el primer bloque temático por número de artículos corresponde también a Ciencias auxiliares, en concreto Epigrafía y Numismática, lo que justificaría que Rgid posea el segundo semiperiodo más alto de la muestra (12.36). Por último, $C d m$ presenta el tercer semiperiodo más alto de la muestra (12.23), e igualmente el volumen empleado trata en un $75 \%$ sobre documentos audiovisuales (aspecto específico dentro de Fuentes de información). 


\section{Conclusiones}

Del análisis precedente podemos concluir, en primer lugar, que empleando una muestra amplia de la literatura científica en Biblioteconomía y Documentación publicada en las revistas de mayor factor de impacto del área (1162 artículos y 41143 referencias válidas), se obtiene un semiperiodo global de 6.91 años en nuestra área de conocimiento para el año 2006, lo que puede considerarse una aproximación razonable sobre el semiperiodo de la literatura científica en Biblioteconomía y Documentación, en este caso advirtiendo la preponderancia cuantitativa de la literatura anglosajona en el cálculo efectuado (905 artículos y 37525 referencias en revistas redactadas en inglés frente a los 257 artículos y 3618 referencias pertenecientes a revistas españolas).

Los datos confirman, en segundo lugar, que el grado de envejecimiento u obsolescencia de la literatura científica en un área de conocimiento dado depende del idioma empleado en la comunicación de los resultados de la investigación [Terrada, Cueva, Añon, 1979; Ruiz Baños, Jiménez-Contreras, 1996]. En el caso concreto de la Biblioteconomía y Documentación, sin embargo, los resultados obtenidos con un corpus mayor (14 revistas en inglés y 14 revistas españolas) y dos únicos idiomas (español e inglés), revelan una diferencia más moderada que los resultados precedentes, al obtenerse un valor del semiperiodo para la literatura anglosajona de 6.91 años y de 6.25 años para la literatura española sobre Biblioteconomía y Documentación en 2006. Ello implica que el envejecimiento de la literatura sobre Documentación en español tiende a ser ligeramente más rápido (las citas tienden a ser un poco más recientes), frente a un envejecimiento levemente más lento en la literatura sobre Documentación en inglés (las citas tienden a ser un poco más antiguas).

Del análisis efectuado se concluye igualmente una mayor variabilidad del semiperiodo en el caso de las revistas españolas sobre Biblioteconomía y Documentación. Las revistas en inglés presentan un rango de 4.56 años (entre un máximo de 9.04 años y un mínimo de 4.48 años), cuando el rango en el que se mueven los semiperiodos de las revistas españolas se eleva a 12.47 años (entre un máximo de 15.48 y un mínimo de 3.01 años). Una explicación razonable de este hecho, en el corpus que nos ocupa, sería la concentración, en algunas revistas españolas y en 2006, de artículos sobre aspectos temáticos muy concretos que tienden a aumentar el semiperiodo (es el caso de Bilduma, con semiperiodo de 15.48, cuya totalidad de artículos en 2006 versa sobre aspectos históricos), o bien la concentración de artículos sobre un aspecto temático muy concreto que tiende a disminuir el semiperiodo (es el caso de Baab, con semiperiodo de 3.01, en cuyo volumen correspondiente 
a 2006, dos terceras partes de los artículos versan sobre Biblioteconomía y cuyo tercio restante versa sobre Profesionales de la información y usuarios, aspectos temáticos ambos que favorecen esta tendencia).

Por último, la muestra empleada aporta indicios suficientes que avalan la hipótesis planteada por Burton y Kebler en 1960 sobre la relación entre semiperiodo y el contenido temático de las revistas. Aunque únicamente sea a nivel de análisis inicial, y a falta de un estudio más profundo que tenga en cuenta un corpus mayor, podemos distinguir tres clases de temas en el área de la Biblioteconomía y Documentación:

- Aspectos temáticos nucleares del área de la Biblioteconomía y Documentación. Se caracterizan porque su presencia mayoritaria favorece que el semiperiodo se mantenga en un rango cercano al promedio del semiperiodo del área. Serían, en consecuencia, los aspectos temáticos principales responsables del semiperiodo obtenido previamente. Destacan en este apartado "Estudios métricos de la información”, “Tecnologías de la información" y "Proceso documental".

- Aspectos temáticos que favorecen la disminución del semiperiodo. Se caracterizan porque su presencia mayoritaria en los artículos de una revista ayuda a que el semiperiodo resultante de dicha revista disminuya. Destacan en este apartado "Profesionales de la información y usuarios", "Biblioteconomía” y "Ciencia de la Documentación”.

- Aspectos temáticos que favorecen el aumento del semiperiodo. Se caracterizan porque su presencia mayoritaria en los artículos de una revista ayuda a que el semiperiodo resultante de dicha revista aumente. Destacan en este apartado "Historia”, "Epigrafía”, "Numismática” y "Fondo antiguo".

Los indicios hallados sobre la relación entre el semiperiodo y los aspectos temáticos abordados en los artículos precisan, sin duda, de un análisis más profundo, no exento de dificultades metodológicas. El primero y más importante consiste en que los resultados que se obtengan dependen de la clasificación temática de la que se parta. En este estudio hemos comprobado, por ejemplo, que el descriptor temático "Ciencias auxiliares" era excesivamente amplio, y englobado aspectos temáticos que favorecían tanto el aumento como la disminución del semiperiodo. En segundo lugar, es necesario decidir cómo tratar la confluencia de diversos aspectos temáticos en un único artículo. En este trabajo se impuso finalmente un único descriptor temático por artículo, con el ánimo de facilitar el estudio presentado, pero somos conscientes de que posiblemente un análisis más profundo que permitiese distinguir 
porcentajes temáticos en cada artículo daría lugar a resultados mucho más concluyentes que los presentados aquí.

Para terminar, cabe resaltar que el análisis efectuado avala la relación entre semiperiodo y aspectos temáticos que ya plantearon Burton y Kebler en 1960, no sólo a nivel de grandes áreas de conocimiento, sino también dentro de cada una de ellas, empleando en nuestro caso diversas categorías temáticas dentro de la Biblioteconomía y Documentación. De confirmarse esta relación en posteriores trabajos, dispondríamos de un método cuantitativo sencillo para comparar la evolución temporal de cada una de dichas categorías temáticas dentro de nuestra área de conocimiento, además de obtener la preponderancia de algunas de dichas categorías frente a otras a lo largo del tiempo. De este modo podríamos, en última instancia, cuantificar los cambios que se fuesen produciendo en nuestra área desde el punto de vista de los contenidos presentes en su literatura científica.

\section{Bibliografía}

Burton, R.E.; Kebler, R.W., "The'half-life' of some scientific and technical literatures", en American Documentation, 1960, vol. XI, pp. 18-22.

CINDOC, Tesauro de Biblioteconomía y Documentación, disponible en: http://thes.cindoc.csic.es/index_BIBLIO_esp.html, consultado: 09-03-2010.

IN-RECS, Biblioteconomía. Revistas ordenadas por Índice de impacto, disponible en: http://ec3.ugr.es/in-recs/Biblioteconomia.htm, consultado: 09-03-2010.

Journal Citation Reports. Cited Half-Life, disponible en: http://admin-apps.isiknowledge.com/JCR/help/h_ctdhl.htm, consultado: 09-03-2010.

Journal Citation Reports, Social Science Edition. Journal Summary List [2005] sorted by impact factor, disponible en: http://sauwok. fecyt.es/admin-apps/JCR/JCR, consultado: 09-03-2010.

Ruiz Baños, R.; Bailón-Moreno, R., "Métodos para medir experimentalmente el envejecimiento de la literatura científica", en Boletín de la Asociación Andaluza de Bibliotecarios, 1997, núm. 46, marzo, pp. $57-75$.

Ruiz Baños, R.; Jiménez-Contreras, E., "Envejecimiento de la literatura científica en documentación. Influencia del origen nacional de las revistas. Estudio de una muestra”, en Revista Española de Documentación Científica, 1996, vol. 19, núm. 1, pp. 39-49. 
Terrada, M.L.; Cueva, A. de la; Añon, R., "La obsolescencia de la información científica en las publicaciones médicas españolas”, en Revista Española de Documentación Científica, 1979, vol. 2, núm. 1, pp. 9-32.

Thomson Reuters. Introducing the impact factor, disponible en: http://homsonreuters.com/products_services/science/academic/impact_factor/, consultado: 09-03-2010. 
